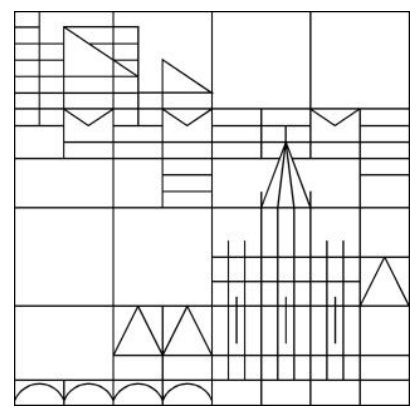

\title{
The Cauchy problem for thermoelastic plates with two temperatures
}

\author{
Reinhard Racke \\ Yoshihiro Ueda
}

Konstanzer Schriften in Mathematik

Nr. 381, März 2019

ISSN 1430-3558 



\title{
THE CAUCHY PROBLEM FOR THERMOELASTIC PLATES WITH TWO TEMPERATURES
}

\author{
REINHARD RACKE AND YOSHIHIRO UEDA
}

\begin{abstract}
We consider the decay rates of solutions to thermoelastic systems in materials where, in contrast to classical thermoelastic models for Kirchhoff type plates, two temperatures are involved, related by an elliptic equation. The arising initial value problems deal with systems of partial differential equations involving Schrödinger like equations, hyperbolic and elliptic equations. Depending on the model - with Fourier or with Cattaneo type heat conduction - we obtain polynomial decay rates without or with regularity loss. This way we obtain another example where the loss of regularity in the Cauchy problem corresponds to the loss of exponential stability in bounded domains. The well-posedness is done using semigroup theory in appropriate space reflecting the different regularity compared to the classical single temperature case, and the (optimal) decay estimates are obtained with sophisticated pointwise estimates in Fourier space.
\end{abstract}

Keywords: thermoelastic plate, Cauchy problem, Fourier and Cattaneo law, asymptotic behavior

MSC 2010: 35B35, 35B40, 35M30, 35Q79, 74F05

\section{INTRODUCTION}

Classical thermoelastic plates of Kirchhoff type modeled by

$$
\begin{aligned}
u_{t t}+b \Delta^{2} u+d \Delta \theta & =0, \\
\theta_{t}+\operatorname{div} q-d \Delta u_{t} & =0, \\
\tau q_{t}+q+\kappa \nabla \theta & =0,
\end{aligned}
$$

for $(u, \theta, q)=(u, \theta, q)(t, x)$ denoting the displacement, the temperature and heat flux for $x \in \mathbb{R}^{n}, t \geq 0$, with $b, d, \kappa>0, \tau \geq 0$, have been discussed in recent years with respect to well-posedness and asymptotic behavior in time (also for bounded and unbounded domains with boundaries, and both for $\tau=0$ and for $\tau>0$.

So-called non-simple materials are modeled by two temperatures, the thermodynamic temperature $\theta$ and the conductive temperature $\psi$, related to each other in the following way, see $[2,3,4,36]$,

$$
\theta=\psi-a \Delta \psi
$$

with a constant $a \geq 0$. The corresponding extension of the classical thermoelastic plate model (1.1) then reads as

$$
\begin{aligned}
u_{t t}+b \Delta^{2} u+d \Delta \theta & =0, \\
\theta_{t}+\operatorname{div} q-d \Delta u_{t} & =0, \\
\tau q_{t}+q+\kappa \nabla \psi & =0,
\end{aligned}
$$


with (1.2). Especially, for $\tau=0,(1.3)$ is rewritten as

$$
\begin{aligned}
& u_{t t}+b \Delta^{2} u+d \Delta \theta=0, \\
& \theta_{t}-\kappa \Delta \psi-d \Delta u_{t}=0,
\end{aligned}
$$

which represents Fourier type heat conduction.

For $a=0$ in (1.2) we recover (1.1). This case was investigated in [31] cf. SaidHouari for the one-dimensional case [33]. A regularity loss in the description of polynomial decay of solutions was proved reflecting the loss of exponential stability in bounded domains known. In this case it is known that one has, for appropriate boundary conditions, exponential stability for the Fourier type heat conduction given by $\tau=0$, while it is not exponentially stable for the Cattaneo (Maxwell) type given for a positive relaxation constant $\tau>0$, see [26, 27, 8].

Here, we shall investigate initial value problems for the case $a>0$. We are first interested in the well-posedness both for $\tau=0$ and for $\tau>0$, which is more delicate in comparison to the case $a=0$, since there will be no regularity gain in the temperature triggered by the differential equations. The main topic will be to investigate the asymptotic behavior as time tends to infinity in terms of polynomial decay rates. The rates will be shown to be optimal, and a loss of regularity will be observed while turning from the Fourier to the Cattaneo model. This way we also contribute a further example where the different heat conduction models, one by Fourier $(\tau=0)$, one by Cattaneo $(\tau>0)$ predict different qualitative behavior. Similar effects are known for the thermoelastic Timoshenko system in one space dimensions. Here, we also have that the system with the Fourier model for heat conduction may show exponential stability in bounded domains (in the case of equal wave speeds of the two wave equations involved), while this property is lost with the Cattaneo model, see Fernańdez Sare \& Racke [9]. Moreover, for the Cauchy problem in $\mathbb{R}^{1}$, one has the same effect, i.e., a regularity-loss phenomenon when changing from Fourier's to Cattaneo's law, see Ide \& Kawashima [12], Ide \& Haramoto \& Kawashima [11], Ueda \& Duan \& Kawashima [35], Said-Houari \& Kasimov [34].

Some further related papers are given as follows:

Case $a=0$ : For bounded domains and for $\tau=0$, there are many results in particular on exponential stability, see for example $[1,13,15,16,17,18,21,22,23]$. For results for the Cauchy problem or in general exterior domains see for example $[5,6,7,22$, $23,31]$. For $\tau>0$, exponential stability in bounded domains is lost $[26,27,8]$, for the Cauchy problem we encounter a regularity loss [31].

Case $a>0$ : As mentioned above, in [28], the bounded domain case was studied, and the exponential stablity was proved for $\tau=0$, while it was shown not to be exponentially stable for $\tau>0$.

The second-order system

$$
\begin{gathered}
u_{t t}-b u_{x x}+d \theta_{x}=0, \\
\theta_{t}+q_{x}+d u_{t x}=0, \\
\tau q_{t}+q+\kappa \psi_{x}=0 \\
\theta-\psi+a \psi_{x x}=0 \\
2
\end{gathered}
$$


in one space dimension in a bounded interval has been studied with respect to exponential stability for $a=0$ [25], the well-posedness was obtained in any space dimension [24]. The non-exponential stability for $\tau>0$ was proved in in [20].

We remark that related nonlinear problems have been discussed in [10, 14, 19, 32].

Our main new contributions are

- First discussion of the fourth-order thermoelastic plate system with two temperatures in all of $\mathbb{R}^{n}$.

- Proof of well-posedness for rather weak regular solutions, both for $\tau=0$ and for $\tau>0$.

- Proof of optimal decay rates for both cases $(\tau=0)$ and $(\tau>0)$

- Demonstration of the loss of regularity while turning from the Fourier model to the Cattaneo one, this way yielding another example for the general picture of the correspondence between "loss of exponential stability in bounded domains" and "loss of regularity for the Cauchy problem".

- Clarification of the role of the heat conduction parameter $\kappa$ in the decay estimates.

The methods used will be essentially sophisticated pointwise estimates of the solutions in Fourier space, and semigroup theory for the well-posedness.

The paper is organized as follows: We start in section 2 proving the well-posedness based on semigroup theory both for $\tau=0$ and for $\tau>0$. In section 3, we treat the case $\tau=0$ and derive the decay estimates using the Fourier transform. Section 4 discusses the decay estimates for the case $\tau>0$.

Throughout the paper, we use standard notation, in particular the Sobolev spaces $L^{p}=L^{p}\left(\mathbb{R}^{n}\right), p \geq 1$, and $H^{s}=W^{s, 2}\left(\mathbb{R}^{n}\right), s \in \mathbb{N}_{0}$, with their associated norms $\|\cdot\|_{L^{p}}$ with abbreviation $\|\cdot\|:=\|\cdot\|_{L^{2}}$, respectively $\|\cdot\|_{H^{s}}$. For the inner product in $L^{2}$ we use the notation $\langle\cdot, \cdot\rangle$. Furthermore, we use the Sobolev norm $\|\cdot\|_{H_{a}^{s}}$ with parameters $\tau$ and $a$ as follows. Let $\tau \geq 0$ and $a \geq 0$ be real numbers, then

$$
\begin{aligned}
\|u\|_{H_{a}^{1}} & :=\left\|\left(1+a|\xi|^{2}\right)^{1 / 2} \hat{u}\right\|_{L^{2}}, \\
\|u\|_{H_{\tau, a}^{3+s}} & :=\left\|\left(1+\tau|\xi|^{2}\right)\left(1+a|\xi|^{2}\right)^{(1+s) / 2} \hat{u}\right\|_{L^{2}},
\end{aligned}
$$

with $s=0,1$, where $\hat{u}$ denotes the Fourier transform of $u$ and $\xi \in \mathbb{R}^{n}$ is the Fourier variable. We observe that $\|\cdot\|_{H_{0}^{s}}=\|\cdot\|_{H_{0,0}^{3+s}}=\|\cdot\|_{L^{2}}$.

\section{WELL-POSEDNESS FOR $\tau \geq 0$}

To discuss the decay estimate of the global solutions in time, we first consider the well-posedness of the systems, both for $\tau=0$ and for $\tau>0$, based on semigroup arguments. Especially, this argument is useful for the system with $\tau>0$ to construct the global solution in time. Regarding the well-posedness of the systems under consideration, we point out, that due to the effects of the two temperatures in the model, the regularity for the temperature(s) is different from that for the case with a single temperature. In the heat equation instead of the Laplace operator, now, a bounded operator appears not triggering the regularity seemingly needed for the main elastic equation for the displacement. As a consequence, a connected regularity is considered. 
We modify the work on the bounded domain case given in our recent paper [28] for the Cauchy problem considered here, and we present the details for the reader's convenience.

2.1. Well-posedness for $\tau=0$. We start proving the well-posedness of the system (1.4) with $\tau=0$, i.e. for (1.4) with the initial data

$$
u(0, x)=u_{0}(x), \quad u_{t}(0, x)=u_{1}(x), \quad \theta(0, x)=\theta_{0}(x)
$$

for $x \in \mathbb{R}^{n}$. We rewrite $(1.2)$ as

$$
\psi=(\operatorname{Id}-a \Delta)^{-1} \theta
$$

where Id denotes the identity operator. Here $(\operatorname{Id}-a \Delta)^{-1}$ denotes the homeomorphism from $L^{2}$ onto $H^{2}$. Then (1.4) with (1.2) can be written as

$$
\begin{gathered}
u_{t t}+\Delta(b \Delta u+d \theta)=0, \\
\theta_{t}-B \theta-d \Delta u_{t}=0,
\end{gathered}
$$

where $B: L^{2} \rightarrow L^{2}$ is a bounded operator defined by $B:=\kappa \Delta(\operatorname{Id}-a \Delta)^{-1}$.

Remark 2.1. The second equation of (2.2) for, essentially, $\theta$ does not trigger any regularity for $\theta$, in contrast to the situation where $a=0$ (only one temperature $\theta=\psi$ ). For $a=0$ we would have the classical operator $B=\kappa \Delta$ on its usual domain. On the other hand, in the first equation of (2.2) one needs, yet formally, $\Delta \theta$. This lack of regularity will be reflected in a lack of separate for regularity for $u$ and $\theta$. We shall have a connected regularity, see below.

The operator $B$ satisfies for (1.2)

$$
\langle B \theta, \theta\rangle=-\kappa\|\nabla \psi\|^{2}-\kappa a\|\Delta \psi\|^{2} \leq 0 .
$$

We transform the system (2.2) into a system of first order in time for $U:=(v, w, \theta)^{T}$ with $v:=u_{t}, w:=\Delta u$, where $T$ denotes the transposed matrix:

$$
U_{t}=\left(\begin{array}{ccc}
0 & -b \Delta & -d \Delta \\
\Delta & 0 & 0 \\
d \Delta & 0 & B
\end{array}\right) U=: A_{f} U, \quad U(0, \cdot)=U^{0}:=\left(u_{1}, \Delta u_{0}, \theta_{0}\right)^{T} .
$$

This formal system with the formal differential symbol $A_{f}$ will be considered as an evolution equation in the associated Hilbert space $\mathcal{H}:=L^{2} \times L^{2} \times L^{2}$ with inner product

$$
\langle U, W\rangle_{\mathcal{H}}:=\left\langle U^{1}, W^{1}\right\rangle+b\left\langle U^{2}, W^{2}\right\rangle+\left\langle U^{3}, W^{3}\right\rangle,
$$

where $U=\left(U^{1}, U^{2}, U^{3}\right)^{T}$ and $W=\left(W^{1}, W^{2}, W^{3}\right)^{T}$. Then our problem is associated with

$$
U_{t}=A U, \quad U(t=0)=U^{0}
$$

where

$$
A: D(A) \subset \mathcal{H} \longrightarrow \mathcal{H}, \quad A U:=A_{f} U,
$$

for $U \in D(A)$ with

$$
D(A):=\left\{U=(v, w, \theta)^{T} \in \underset{4}{\mathcal{H}} \mid v \in H^{2}, \Delta(b w+d \theta) \in L^{2}\right\} .
$$


In the definition of $D(A)$, the problem of the missing (separate) regularity for $\theta$ is reflected. cp. Remark 2.1. One just has the combined regularity $\Delta(b w+d \theta) \in L^{2}$, not writing $\Delta w, \Delta \theta \in L^{2}$, and this way $A_{f} U$ has to be interpreted.

As usual, $\Delta(b w+d \theta) \in L^{2}$ means

$$
\exists h \in L^{2}, \forall \phi \in C_{0}^{\infty}:\langle b w+d \theta, \Delta \phi\rangle=\langle h, \phi\rangle .
$$

We remark that the difference to the bounded domain case considered in [28] lies in the choice of the transformation by $U$ and the choice of the space $\mathcal{H}$. This modification is necessary essentially because in all of $\mathbb{R}^{n}$, the set $H^{2}$ is no longer a Banach space under the norm $\|\Delta \cdot\|$, in contrast to the situation in bounded domains considered in $[28]$.

We will show that $A$ generates a contraction semigroup.

Lemma 2.2. $D(A)$ is dense in $\mathcal{H}$, and for $U \in D(A)$ with (1.2), we have the dissipativity of $A$ that

$$
\operatorname{Re}\langle A U, U\rangle_{\mathcal{H}}=-\kappa\|\nabla \psi\|^{2}-\kappa a\|\Delta \psi\|^{2} \leq 0 .
$$

Proof. $\left(C_{0}^{\infty}\right)^{3} \subset D(A)$ is dense in $\mathcal{H}$. Furthermore, the computation

$$
\begin{aligned}
\operatorname{Re}\langle A U, U\rangle_{\mathcal{H}} & =\operatorname{Re}(\langle-\Delta(b w+d \theta), v\rangle+b\langle\Delta v, w\rangle+\langle d \Delta v+B \theta, \theta\rangle) \\
& =\langle B \theta, \theta\rangle=-\kappa\|\nabla \psi\|^{2}-\kappa a\|\Delta \psi\|^{2} \leq 0
\end{aligned}
$$

gives the proof.

Lemma 2.3. The range of $\mathrm{Id}-A$ equals $\mathcal{H}$.

Proof. (Id $-A) U=F$ is, for given $F=\left(F^{1}, F^{2}, F^{3}\right)^{T} \in \mathcal{H}$, equivalent to finding $U \in D(A)$ solving

$$
\left\{\begin{aligned}
U^{1}+\Delta\left(b U^{2}+d U^{3}\right) & =F^{1}, \\
U^{2}-\Delta U^{1} & =F^{2} \\
U^{3}-d \Delta U^{1}-B U^{3} & =F^{3} .
\end{aligned}\right.
$$

Here, $U^{2}:=\Delta U^{1}+F^{2}$ will be given if we find $\left(U^{1}, U^{3}\right)$ satisfying

$$
\left\{\begin{aligned}
U^{1}+\Delta\left(b \Delta U^{1}+b F^{2}+d U^{3}\right) & =F^{1}, \\
U^{3}-d \Delta U^{1}-B U^{3} & =F^{3},
\end{aligned}\right.
$$

with $U \in D(A)$. For this purpose we consider the sesquilinear form

$$
\beta: \mathcal{K}:=H^{2} \times L^{2} \longrightarrow \mathbb{C},
$$

where $H^{2}$ is equipped with the usual $H^{2}$-norm, and

$$
\begin{gathered}
\beta\left(\left(U^{1}, U^{3}\right),\left(W^{1}, W^{3}\right)\right):=\left\langle U^{1}, W^{1}\right\rangle+\left\langle b \Delta U^{1}+d U^{3}, \Delta W^{1}\right\rangle+\left\langle U^{3}, W^{3}\right\rangle \\
-\left\langle d \Delta U^{1}, W^{3}\right\rangle-\left\langle B U^{3}, W^{3}\right\rangle .
\end{gathered}
$$

The variational problem associated to $(2.5)$ is to find a (unique) $\left(U^{1}, U^{3}\right) \in \mathcal{K}$ satisfying for all $\left(W^{1}, W^{3}\right) \in \mathcal{K}$

$$
\beta\left(\left(U^{1}, U^{3}\right),\left(W^{1}, W^{3}\right)\right)=\left\langle F^{1}, W^{1}\right\rangle+\left\langle F^{3}, W^{3}\right\rangle-\left\langle b F^{2}, \Delta W^{1}\right\rangle .
$$


The solvability of the variational problem follows from the theorem of Lax and Milgram, observing

$$
\left|\beta\left(\left(U^{1}, U^{3}\right),\left(W^{1}, W^{3}\right)\right)\right| \leq C\left\|\left(U^{1}, U^{3}\right)\right\|_{\mathcal{K}}\left\|\left(W^{1}, W^{3}\right)\right\|_{\mathcal{K}}
$$

with some positive constant $C>0$, and

$$
\begin{aligned}
\operatorname{Re} \beta\left(\left(U^{1}, U^{3}\right),\left(U^{1}, U^{3}\right)\right) & =\left\|U^{1}\right\|^{2}+b\left\|\Delta U^{1}\right\|^{2}+\left\|U^{3}\right\|^{2}-\left\langle B U^{3}, U^{3}\right\rangle \\
& \geq c\left\|\left(U^{1}, U^{3}\right)\right\|_{\mathcal{K}}^{2}
\end{aligned}
$$

with some positive constant $c>0$, by elliptic regularity.

By the Lumer-Phillips theorem we conclude the well-posedness:

Theorem 2.4. A generates a contraction semigroup, and, for any $U^{0} \in D(A)$, there is a unique solution $U$ to (2.3) satisfying

$$
U \in C^{1}([0, \infty), \mathcal{H}) \cap C^{0}([0, \infty), D(A)) .
$$

2.2. Well-posedness for $\tau>0$. The model (1.3), (1.2) for thermoelastic plates of Kirchhoff type with two temperatures under the Cattaneo law will now be shown to be well-posed. The well-posedness requires the choice of suitable representations of the solutions and corresponding phase spaces. The regularity issue is even more complicated due to the fact that the heat flux is not immediately of the same regularity as the gradient of the temperature $\psi$, as it was in the case of the Fourier model discussed in the previous section. The issue of only combined regularity for $(u, \theta, q)$ only, in contrast to separate regularity for each of $u, \theta, q$, comes up again requiring the right spaces and domains of operators.

We consider the Cauchy problem (1.3), (1.2) with initial data

$$
u(0, x)=u_{0}(x), \quad u_{t}(0, x)=u_{1}(x), \quad \theta(0, x)=\theta_{0}(x), \quad q(0, x)=q_{0}(x)
$$

for $x \in \mathbb{R}^{n}$. Defining $v:=u_{t}, w:=\sqrt{b} \Delta u$ we obtain from (1.3), (1.2)

$$
\begin{aligned}
v_{t}+\sqrt{b} \Delta w+d \Delta \theta & =0, \\
w_{t}-\sqrt{b} \Delta v & =0, \\
\theta_{t}+\operatorname{div} q-d \Delta v & =0, \\
\tau q_{t}+\nabla B_{1} \theta+q & =0,
\end{aligned}
$$

where $B_{1}$ denotes the bounded operator $B_{1}: L^{2} \rightarrow H^{2}, B_{1}:=\kappa(\operatorname{Id}-a \Delta)^{-1}$. Let $U:=(v, w, \theta, q)^{T}$. Then

$$
\begin{gathered}
U_{t}=\left(\begin{array}{cccc}
0 & -\sqrt{b} \Delta & -d \Delta & 0 \\
\sqrt{b} \Delta & 0 & 0 & 0 \\
d \Delta & 0 & 0 & -\operatorname{div} \\
0 & 0 & -\frac{1}{\tau} \nabla B_{1} & -\frac{1}{\tau}
\end{array}\right) U=: A_{1, f} U \\
U(0, \cdot)=U^{0}:=\left(u_{1}, \sqrt{b} \Delta u_{0}, \theta_{0}, q_{0}\right)^{T} \\
6
\end{gathered}
$$


System (2.7), (2.8) will be considered as an evolution equation in the associated Hilbert space $\mathcal{H}_{1}:=L^{2} \times L^{2} \times L^{2} \times \mathcal{D}$, where $\mathcal{D}:=\left\{q \in L^{2} \mid \operatorname{div} q \in L^{2}\right\}$, with inner product

$$
\langle U, W\rangle_{\mathcal{H}_{1}}:=\left\langle U^{1}, W^{1}\right\rangle+\left\langle U^{2}, W^{2}\right\rangle+\frac{1}{\tau}\left\langle U^{3}, B_{1} W^{3}\right\rangle+\left\langle U^{4}, W^{4}\right\rangle+\left\langle\operatorname{div} U^{4}, \operatorname{div} W^{4}\right\rangle,
$$

where $U=\left(U^{1}, U^{2}, U^{3}, U^{4}\right)^{T}$ and $W=\left(W^{1}, W^{2}, W^{3}, W^{4}\right)^{T}$. Then we solve

$$
U_{t}=A_{1} U, \quad U(t=0)=U^{0},
$$

where

$$
A_{1}: D\left(A_{1}\right) \subset \mathcal{H}_{1} \longrightarrow \mathcal{H}_{1}, \quad A_{1} U:=A_{1, f} U,
$$

for $U \in D\left(A_{1}\right)$ with

$$
D\left(A_{1}\right):=\left\{U=(v, w, \theta, q)^{T} \in \mathcal{H}_{1} \mid v \in H^{2}, \Delta(\sqrt{b} w+d \theta) \in L^{2}\right\} .
$$

In the definition of $D\left(A_{1}\right)$, the problem of the missing (separate) regularity is reflected again, cf. the previous subsection. Again we show that $A_{1}$ generates a $C_{0}$-semigroup. For this purpose we write

$$
A_{1}=\underbrace{\left(\begin{array}{cccc}
0 & -\sqrt{b} \Delta & -d \Delta & 0 \\
\sqrt{b} \Delta & 0 & 0 & 0 \\
d \Delta & 0 & 0 & 0 \\
0 & 0 & 0 & 0
\end{array}\right)}_{=: A_{11}}+\underbrace{\left(\begin{array}{cccc}
0 & 0 & 0 & 0 \\
0 & 0 & 0 & 0 \\
0 & 0 & 0 & -\operatorname{div} \\
0 & 0 & -\frac{1}{\tau} \nabla B_{1} & -\frac{1}{\tau}
\end{array}\right)}_{=: A_{12}} .
$$

The operator $A_{12}: \mathcal{H}_{1} \rightarrow \mathcal{H}_{1}$ is bounded, and for

$$
A_{11}: D\left(A_{11}\right):=D\left(A_{1}\right) \subset \mathcal{H}_{1} \longrightarrow \mathcal{H}_{1}
$$

we have the following fact.

Lemma 2.5. (i) $D\left(A_{11}\right)$ is dense in $\mathcal{H}_{1}$, and $A_{11}$ is dissipative,

$$
\operatorname{Re}\left\langle A_{11} U, U\right\rangle_{\mathcal{H}_{1}}=0 .
$$

(ii) The range of $\mathrm{Id}-A_{11}$ equals $\mathcal{H}_{1}$.

Proof. (i) is easy again, and to find $U=\left(U^{1}, U^{2}, U^{3}, U^{4}\right)^{T} \in D\left(A_{1}\right)$ satisfying (Id $\left.A_{11}\right) U=F$, for given $F=\left(F^{1}, F^{2}, F^{3}, F^{4}\right)^{T} \in \mathcal{H}_{1}$, we may argue as in the proof of Lemma 2.3, now eliminating first $U^{2}, U^{3}$ and $U^{4}$ as follows. $\left(\operatorname{Id}-A_{11}\right) U=F$ is equivalent to

$$
\left\{\begin{aligned}
U^{1}+\Delta\left(\sqrt{b} U^{2}+d U^{3}\right) & =F^{1}, \\
U^{2}-\sqrt{b} \Delta U^{1} & =F^{2}, \\
U^{3}-d \Delta U^{1} & =F^{3}, \\
U^{4} & =F^{4} .
\end{aligned}\right.
$$

After having found the appropriate $U^{1}$, we may take $U^{2}:=\sqrt{b} \Delta U^{1}+F^{2}, U^{3}:=$ $d \Delta U^{1}+F^{3}$ and $U^{4}:=F^{4}$. For $U^{1}$ we have to solve

$$
U^{1}+\Delta\left(b \Delta U^{1}+\sqrt{b} F^{2}+d F^{3}+d^{2} \Delta U^{1}\right)=F^{1} .
$$

This is equivalent to finding $U^{1} \in H^{2}$ satisfying for all $W^{1} \in H^{2}$

$$
\left\langle U^{1}, W^{1}\right\rangle+\left\langle\left(b+d^{2}\right) \Delta U^{1}+\underset{7}{\sqrt{b}} F^{2}+d F^{3}, \Delta W^{1}\right\rangle=\left\langle F^{1}, W^{1}\right\rangle .
$$


Inspired by this equation, we define the bilinear form $\beta_{11}: \mathcal{K}_{1}:=H^{2} \times H^{2} \rightarrow \mathbb{C}$ with

$$
\beta_{11}\left(U^{1}, W^{1}\right):=\left\langle U^{1}, W^{1}\right\rangle+\left\langle\left(b+d^{2}\right) \Delta U^{1}, \Delta W^{1}\right\rangle
$$

and $\mathcal{F}: H^{2} \rightarrow \mathbb{C}$ with

$$
\mathcal{F}\left(W^{1}\right):=\left\langle F_{1}, W^{1}\right\rangle-\left\langle\sqrt{b} F^{2}+d F^{3}, \Delta W^{1}\right\rangle .
$$

Then (2.10) is described as $\beta_{11}\left(U^{1}, W^{1}\right)=\mathcal{F}\left(W^{1}\right)$. Here, $\mathcal{F}$ is a bounded linear functional on $H^{2}$. Furthermore, the bilinear form $\beta_{11}$ satisfies

$$
\begin{aligned}
\left|\beta_{11}\left(U^{1}, W^{1}\right)\right| & \leq C_{1}\left\|U^{1}\right\|_{H^{2}}\left\|W^{1}\right\|_{H^{2}}, \\
\beta_{11}\left(U^{1}, U^{1}\right) & =\left\langle U^{1}, U^{1}\right\rangle+\left\langle\left(b+d^{2}\right) \Delta U^{1}, \Delta U^{1}\right\rangle \geq c_{1}\left\|U^{1}\right\|_{H^{2}}^{2},
\end{aligned}
$$

for some positive constants and $C_{1}$ and $c_{1}$, so we may use the theorem of Lax and Milgram to find the solution $U^{1} \in H^{2}$.

As a consequence we obtain the well-posedness of (2.9) in the following result.

Theorem 2.6. $A_{1}$ generates a $C_{0}$-semigroup, and, for any $U^{0} \in D\left(A_{1}\right)$, there is a unique solution $U$ to (2.9) satisfying

$$
U \in C^{1}\left([0, \infty), \mathcal{H}_{1}\right) \cap C^{0}\left([0, \infty), D\left(A_{1}\right)\right) .
$$

\section{Decay estimates for Fourier type heat CONDUCtion}

In this section, we consider the case $\tau=0$, i.e. system (1.4), (1.2), with initial data (2.1). The purpose of this section is to derive the optimal decay estimates for the global solutions in time.

3.1. Decay estimates $(\tau=0)$. To derive a representation of the solution and to get the decay estimates for the solutions, we introduce new functions $v:=u_{t}, w:=\sqrt{b} \Delta u$ for (1.4). Then our problem reads as

$$
\begin{aligned}
v_{t}+\sqrt{b} \Delta w+d \Delta \theta & =0, \\
w_{t}-\sqrt{b} \Delta v & =0 \\
\theta_{t}-\kappa \Delta \psi-d \Delta v & =0
\end{aligned}
$$

Furthermore, (3.1) with (1.2) leads to

$$
(I-a \Delta) U_{t}-\Delta B_{1} U-(I-a \Delta) \Delta B_{2} U=0,
$$

where $U:=(v, w, \theta)^{T}$ and

$$
B_{1}:=\left(\begin{array}{ccc}
0 & 0 & 0 \\
0 & 0 & 0 \\
0 & 0 & \kappa
\end{array}\right), \quad B_{2}:=\left(\begin{array}{ccc}
0 & -\sqrt{b} & -d \\
\sqrt{b} & 0 & 0 \\
d & 0 & 0
\end{array}\right) .
$$

Applying the Fourier transform to (3.2), we have

$$
\hat{U}_{t}+\frac{|\xi|^{2}}{1+a|\xi|^{2}} B_{1} \hat{U}+|\xi|^{2} B_{2} \hat{U}=0 .
$$

The is can be solved, and the solution of (3.3) can be written as

$$
\hat{U}(t, \xi)=e^{t \hat{\Phi}(i \xi)} \hat{U}_{0}(\xi)
$$


where $U_{0}:=\left(v_{0}, w_{0}, \theta_{0}\right)^{T}$ with $v_{0}:=u_{1}$ and $w_{0}:=\Delta u_{0}$, and

$$
\hat{\Phi}(i \xi):=-\left(\frac{|\xi|^{2}}{1+a|\xi|^{2}} B_{1}+|\xi|^{2} B_{2}\right)=-\left(\begin{array}{ccc}
0 & -\sqrt{b}|\xi|^{2} & -d|\xi|^{2} \\
\sqrt{b}|\xi|^{2} & 0 & 0 \\
d|\xi|^{2} & 0 & \kappa|\xi|^{2} /\left(1+a|\xi|^{2}\right)
\end{array}\right)
$$

Therefore, the semigroup $e^{t \Phi}$ associated with the system (3.2) is given by the formula

$$
e^{t \Phi} \varphi:=\mathcal{F}^{-1}\left[e^{t \hat{\Phi}(i \xi)} \hat{\varphi}(\xi)\right] .
$$

Now, our purpose is to derive the property of the solution operator $e^{t \Phi}$. The result on the decay estimates for (3.2) is stated as follows.

Theorem 3.1. Let $e^{t \Phi}$ be the semigroup associated with the system (3.2) defined by (3.6). Then the following decay estimates hold for $1 \leq p \leq 2$ and $k \geq 0$.

$$
\left\|\partial_{x}^{k} e^{t \Phi} \varphi\right\|_{L^{2}} \leq C_{0}\left(1+\nu_{a} t\right)^{-\frac{n}{2}\left(\frac{1}{p}-\frac{1}{2}\right)-\frac{k}{2}}\|\varphi\|_{L^{p}}+\sqrt{3} e^{-\frac{\nu_{a}}{2} t}\left\|\partial_{x}^{k} \varphi\right\|_{L^{2}},
$$

where

$$
\nu_{a}:=\frac{\nu}{1+a}, \quad \nu:=\frac{b d^{2} \kappa}{12\left(8\left(b+d^{2}\right)^{2}+b \kappa^{2}\right)},
$$

and $C_{0}$ is a certain positive constant depends on only $p$ and $k$.

The key of the proof of Theorem 3.1 is to get the pointwise estimate of the operator $e^{t \Phi}$ in Fourier space, which is stated as follows.

Proposition 3.2. Let $\hat{\Phi}(i \xi)$ be the matrix defined in (3.5). Then the corresponding matrix exponential $e^{t \hat{\Phi}(i \xi)}$ satisfies the following pointwise estimate

$$
\left|e^{t \hat{\Phi}(i \xi)}\right| \leq \sqrt{3} e^{-\frac{1}{2} \rho(\xi) t}
$$

for $t \geq 0$ and $\xi \in \mathbb{R}^{n}$, where

$$
\rho(\xi):=\frac{\nu|\xi|^{2}}{1+a|\xi|^{2}},
$$

and $\nu$ is defined in Theorem 3.1.

Proof. We first derive the basic energy equation for the system (3.2). Taking the inner product (3.2) with $\hat{U}$, and taking real parts in the resulting equality, we have

$$
\frac{1}{2}\left(1+a|\xi|^{2}\right) \frac{\partial}{\partial t}|\hat{U}|^{2}+\kappa|\xi|^{2}|\hat{\theta}|^{2}=0 .
$$

Next, we construct the dissipation terms. System (3.2) gives us

$$
\begin{aligned}
\hat{v}_{t}-\sqrt{b}|\xi|^{2} \hat{w}-d|\xi|^{2} \hat{\theta} & =0, \\
\hat{w}_{t}+\sqrt{b}|\xi|^{2} \hat{v} & =0, \\
\hat{\theta}_{t}+\kappa|\xi|^{2} \hat{\psi}+d|\xi|^{2} \hat{v} & =0,
\end{aligned}
$$

with $\hat{\theta}=\left(1+a|\xi|^{2}\right) \hat{\psi}$. We multiply the first and second equations in (3.11) by $-\overline{\hat{w}}$ and $-\overline{\hat{v}}$, respectively. Then, combining the resulting equations and taking real parts, we obtain

$$
-\frac{\partial}{\partial t} \operatorname{Re}(\hat{v} \overline{\hat{w}})+\sqrt{b}|\xi|^{2}\left(|\hat{w}|^{2}-|\hat{v}|^{2}\right)+d|\xi|^{2} \operatorname{Re}(\hat{w} \overline{\hat{\theta}})=0 .
$$


Especially, using the Hölder inequality, we get

$$
-\sqrt{b} \frac{\partial}{\partial t} \operatorname{Re}(\hat{v} \overline{\hat{w}})+\frac{b}{2}|\xi|^{2}|\hat{w}|^{2}-b|\xi|^{2}|\hat{v}|^{2}-\frac{d^{2}}{2}|\xi|^{2}|\hat{\theta}|^{2} \leq 0 .
$$

Furthermore, we multiply the first and third equations in (3.11) by $\overline{\hat{\theta}}$ and $\overline{\hat{v}}$, respectively. Then we have

$$
\frac{\partial}{\partial t} \operatorname{Re}(\hat{v} \overline{\hat{\theta}})+d|\xi|^{2}\left(|\hat{v}|^{2}-|\hat{\theta}|^{2}\right)+\kappa|\xi|^{2} \operatorname{Re}(\hat{v} \overline{\hat{\psi}})-\sqrt{b}|\xi|^{2} \operatorname{Re}(\hat{w} \overline{\hat{\theta}})=0 .
$$

Similarly as before, we obtain

$$
d \frac{\partial}{\partial t} \operatorname{Re}(\hat{v} \hat{\hat{\theta}})+\frac{d^{2}}{2}|\xi|^{2}|\hat{v}|^{2}-d^{2}|\xi|^{2}|\hat{\theta}|^{2}-\frac{\kappa^{2}}{2}|\xi|^{2}|\hat{\psi}|^{2}-d \sqrt{b}|\xi|^{2} \operatorname{Re}(\hat{w} \overline{\hat{\theta}}) \leq 0 .
$$

Our next step is to combine the above inequalities to construct the energy estimate. First, computing $d^{2} / 4 \times(3.12)+b \times(3.13)$, we get

$$
\begin{aligned}
\frac{\partial}{\partial t}\{b & \left.d \operatorname{Re}(\hat{v} \overline{\hat{\theta}})-\frac{d^{2} \sqrt{b}}{4} \operatorname{Re}(\hat{v} \overline{\hat{w}})\right\}+\frac{b d^{2}}{4}|\xi|^{2}\left(|\hat{v}|^{2}+\frac{1}{2}|\hat{w}|^{2}\right) \\
& -d^{2}\left(b+\frac{d^{2}}{8}\right)|\xi|^{2}|\hat{\theta}|^{2}-\frac{b \kappa^{2}}{2}|\xi|^{2}|\hat{\psi}|^{2}+b d \sqrt{b}|\xi|^{2} \operatorname{Re}(\hat{w} \overline{\hat{\theta}}) \leq 0 .
\end{aligned}
$$

Here, using

$$
b d \sqrt{b}|\hat{w}||\hat{\theta}| \leq \frac{b d^{2}}{16}|\hat{w}|^{2}+4 b^{2}|\hat{\theta}|^{2}
$$

we obtain

$$
\begin{aligned}
\frac{\partial}{\partial t}\{b & \left.d \operatorname{Re}(\hat{v} \hat{\hat{\theta}})-\frac{d^{2} \sqrt{b}}{4} \operatorname{Re}(\hat{v} \overline{\hat{w}})\right\}+\frac{b d^{2}}{4}|\xi|^{2}\left(|\hat{v}|^{2}+\frac{1}{4}|\hat{w}|^{2}\right) \\
- & d^{2}\left(b+\frac{d^{2}}{8}\right)|\xi|^{2}|\hat{\theta}|^{2}-\frac{b \kappa^{2}}{2}|\xi|^{2}|\hat{\psi}|^{2}-4 b^{2}|\xi|^{2}|\hat{\theta}|^{2} \leq 0 .
\end{aligned}
$$

Especially, $\hat{\theta}=\left(1+a|\xi|^{2}\right) \hat{\psi}$ gives us

$$
\frac{\partial}{\partial t}\left\{b d \operatorname{Re}(\hat{v} \hat{\hat{\theta}})-\frac{d^{2} \sqrt{b}}{4} \operatorname{Re}(\hat{v} \overline{\hat{w}})\right\}+\frac{b d^{2}}{4}|\xi|^{2}\left(|\hat{v}|^{2}+\frac{1}{4}|\hat{w}|^{2}\right)-4 g_{0}|\xi|^{2}|\hat{\theta}|^{2} \leq 0,
$$

where

$$
g_{0}:=\left(b+d^{2}\right)^{2}+\frac{b \kappa^{2}}{8} .
$$

Therefore, calculating $g_{0} \times(3.10)+\kappa / 8 \times(3.14)$ yields

$$
\frac{\partial}{\partial t} E_{0}(t, \xi)+\frac{b d^{2} \kappa}{32}|\xi|^{2}\left(|\hat{v}|^{2}+\frac{1}{4}|\hat{w}|^{2}\right)+\frac{\kappa}{2} g_{0}|\xi|^{2}|\hat{\theta}|^{2} \leq 0,
$$

where we define

$$
E_{0}(t, \xi):=\frac{1}{2} g_{0}\left(1+a|\xi|^{2}\right)|\hat{U}|^{2}+\frac{\kappa}{8}\left(b d \operatorname{Re}(\hat{v} \overline{\hat{\theta}})-\frac{d^{2} \sqrt{b}}{4} \operatorname{Re}(\hat{v} \overline{\hat{w}})\right) .
$$

Here, we easily obtain from

$$
d^{2} \kappa \sqrt{b}|\hat{v}||\hat{w}| \leq \frac{1}{2}\left(b \kappa^{2}|\hat{v}|^{2}+d^{4}|\hat{w}|^{2}\right), \quad d \kappa|\hat{v}||\hat{\theta}| \leq d^{2}|\hat{v}|^{2}+\frac{\kappa^{2}}{4}|\hat{\theta}|^{2}
$$


the estimate

$$
\frac{1}{4} g_{0}\left(1+a|\xi|^{2}\right)|\hat{U}|^{2} \leq E_{0}(t, \xi) \leq \frac{3}{4} g_{0}\left(1+a|\xi|^{2}\right)|\hat{U}|^{2}
$$

Therefore, integrating (3.15) over t and applying (3.16) to the resulting estimate, we get

$$
|\hat{U}(t, \xi)|^{2}+\int_{0}^{t} \frac{\kappa|\xi|^{2}}{1+a|\xi|^{2}}\left\{\frac{b d^{2}}{8 g_{0}}\left(\left|\hat{v}\left(t^{\prime}, \xi\right)\right|^{2}+\frac{1}{4}\left|\hat{w}\left(t^{\prime}, \xi\right)\right|^{2}\right)+2\left|\hat{\theta}\left(t^{\prime}, \xi\right)\right|^{2}\right\} d t^{\prime} \leq 3\left|\hat{U}_{0}(\xi)\right|^{2}
$$

hence

$$
\left\|\partial_{x}^{k} U(t)\right\|_{H_{a}^{1}}^{2}+\frac{b d^{2} \kappa}{32 g_{0}} \int_{0}^{t}\left\|\partial_{x}^{k+1} U\left(t^{\prime}\right)\right\|_{L^{2}}^{2} d t^{\prime} \leq 3\left\|\partial_{x}^{k} U_{0}\right\|_{H_{a}^{1}}^{2},
$$

for $k \geq 0$. Furthermore, (3.15) and

$$
\frac{b d^{2} \kappa}{32}|\xi|^{2}\left(|\hat{v}|^{2}+\frac{1}{4}|\hat{w}|^{2}\right)+\frac{\kappa}{2} g_{0}|\xi|^{2}|\hat{\theta}|^{2} \geq \frac{b d^{2} \kappa}{128}|\xi|^{2}|\hat{U}|^{2} \geq \frac{b d^{2} \kappa}{96 g_{0}} \frac{|\xi|^{2}}{1+a|\xi|^{2}} E_{0}(t, \xi),
$$

which comes from (3.16), gives us

$$
\frac{\partial}{\partial t} E_{0}(t, \xi)+\frac{b d^{2} \kappa}{96 g_{0}} \frac{|\xi|^{2}}{1+a|\xi|^{2}} E_{0}(t, \xi) \leq 0 .
$$

Thus, we obtain $E_{0}(t, \xi) \leq e^{-\rho(\xi) t} E_{0}(0, \xi)$, where $\rho(\xi)$ is defined in Proposition 3.2. This means $|\hat{U}(t, \xi)| \leq \sqrt{3} e^{-\rho(\xi) t / 2}\left|\hat{U}_{0}(\xi)\right|$, and (3.4) gives the the desired pointwise estimate (3.8).

Proof of Theorem 3.1. We use the Hausdorff-Young inequality, $\|\hat{f}\|_{L^{p^{\prime}}} \leq(2 \pi)^{n / p^{\prime}}\|f\|_{L^{p}}$ for $1 \leq p \leq 2$ and $1 / p+1 / p^{\prime}=1$.

By (3.8), we have

$$
\left\|\partial_{x}^{k} e^{t \Phi} \varphi\right\|^{2} \leq 3 \int_{|\xi| \leq 1}|\xi|^{2 k} e^{-\rho(\xi) t}|\hat{\varphi}(\xi)|^{2} d \xi+3 \int_{|\xi| \geq 1}|\xi|^{2 k} e^{-\rho(\xi) t}|\hat{\varphi}(\xi)|^{2} d \xi=: I_{1}+I_{2}
$$

For the case $|\xi| \leq 1$, we compute $\rho(\xi) \geq \nu_{a}|\xi|^{2}$, and then

$$
\begin{aligned}
I_{1} & \leq 3 \int_{|\xi| \leq 1}|\xi|^{2 k} e^{-\nu_{a}|\xi|^{2} t}|\hat{\varphi}(\xi)|^{2} d \xi \\
& \leq 3\left\||\xi|^{2 k} e^{-\nu_{a}|\xi|^{2} t}\right\|_{L^{\sigma}(|\xi| \leq 1)}\|\hat{\varphi}\|_{L^{p^{\prime}(|\xi| \leq 1)}}^{2} \leq C_{0}^{2}\left(1+\nu_{a} t\right)^{-n /(2 \sigma)-k}\|\varphi\|_{L^{p}}^{2}
\end{aligned}
$$

for $1 / \sigma+2 / p^{\prime}=1,1 / p+1 / p^{\prime}=1$ and $1 \leq p \leq 2$, where we used the Hölder inequality and the Hausdorff-Young inequality. Here we note that $1 / \sigma=2 / p-1$, and we then arrive at

$$
I_{1} \leq C_{0}^{2}\left(1+\nu_{a} t\right)^{-n\left(\frac{1}{p}-\frac{1}{2}\right)-k}\|\varphi\|_{L^{p}}^{2}
$$

for $1 \leq p \leq 2$, where $C_{0}$ is a positive constant depends on only $p$ and $k$. For the case $|\xi| \geq 1$, we calculate $\rho(\xi) \geq \nu_{a}$ and

$$
I_{2} \leq 3 e^{-\nu_{a} t} \int_{|\xi| \geq 1}|\xi|^{2 k}|\hat{\varphi}(\xi)|^{2} d \xi \leq 3 e^{-\nu_{a} t}\left\|\partial_{x}^{k} \varphi\right\|_{L^{2}}^{2}
$$

Consequently, we get

$$
\left\|\partial_{x}^{k} e^{t \Phi} \varphi\right\|^{2} \leq C_{0}^{2}\left(1+\nu_{a} t\right)^{-n\left(\frac{1}{p}-\frac{1}{2}\right)-k}\|\varphi\|_{L^{p}}^{2}+3 e^{-\nu_{a} t}\left\|\partial_{x}^{k} \varphi\right\|_{L^{2}}^{2}
$$

and arrive at the desired decay estimate (3.7). 
3.2. Optimality of the decay estimates $(\tau=0)$. At the end of this section, we investigate the optimality of the pointwise estimates in Theorem 3.1. To this end, we consider the characteristic equation $\operatorname{det}(\lambda I-\hat{\Phi}(i \xi))=0$ for the system (3.2), which is equivalent to

$$
\left(1+a|\xi|^{2}\right) \lambda^{3}+\kappa|\xi|^{2} \lambda^{2}+\left(b+d^{2}\right)\left(1+a|\xi|^{2}\right)|\xi|^{4} \lambda+b \kappa|\xi|^{6}=0 .
$$

We consider the asymptotic expansion of $\lambda=\lambda(i \xi)$ for $|\xi| \rightarrow 0$ and for $|\xi| \rightarrow \infty$. These expansions essentially determine the asymptotic behavior of solutions.

We first consider the asymptotic expansion for $|\xi| \rightarrow 0$ :

$$
\lambda(i \xi)=\sum_{k=0}^{\infty} \alpha_{k}|\xi|^{k} .
$$

Substituting (3.18) into (3.17), we have identities with respect to the order of $|\xi|$. From the zeroth order and third order identities, we obtain $\alpha_{0}=0$ and $\alpha_{1}=0$, respectively. Furthermore, the sixth order identity gives $f\left(\alpha_{2}\right)=0$, where

$$
f(z):=z^{3}+\kappa z^{2}+\left(b+d^{2}\right) z+b \kappa .
$$

Let $z_{j}$ with $j=1,2,3$ be solutions for $f(z)=0$. Then these solutions satisfy $z_{1}+$ $z_{2}+z_{3}=-\kappa$. Since $f(0)=b \kappa>0$ and $f(-\kappa)=-d^{2} \kappa<0$, we get $\operatorname{Re}\left(z_{j}\right)<0$ for $j=1,2,3$. Thus, we conclude that the solutions for (3.17) satisfy

$$
\lambda_{j}(i \xi)=z_{j}|\xi|^{2}+O\left(|\xi|^{4}\right)
$$

for $j=1,2,3$.

Next, we consider the asymptotic expansion for $|\xi| \rightarrow \infty$. To this end, we take $\lambda=|\xi|^{2} \nu$ in (3.17) and obtain

$$
\left(a+|\xi|^{-2}\right) \nu^{3}+\kappa|\xi|^{-2} \nu^{2}+\left(b+d^{2}\right)\left(a+|\xi|^{-2}\right) \nu+b \kappa|\xi|^{-2}=0 .
$$

We substitute

$$
\nu(i \xi)=\sum_{k=0}^{\infty} \beta_{k}|\xi|^{-k}
$$

into (3.21). Then the zeroth order identity gives $\beta_{0}=0, \pm \sqrt{b+d^{2}} i$. Furthermore, this yields $\beta_{1}=0, \beta_{3}=0$ and

$$
\beta_{2}=-\frac{\kappa\left(\beta_{0}^{2}+b\right)}{a\left(3 \beta_{0}^{2}+b+d^{2}\right)} .
$$

Therefore, we derive

$$
\begin{aligned}
& \lambda_{j}(i \xi)= \pm \sqrt{b+d^{2}} i|\xi|^{2}-\frac{d^{2} \kappa}{2 a\left(b+d^{2}\right)}+O\left(|\xi|^{-2}\right) \\
& \lambda_{3}(i \xi)=-\frac{b \kappa}{a\left(b+d^{2}\right)}+O\left(|\xi|^{-2}\right)
\end{aligned}
$$

for $j=1,2$.

Consequently, the asymptotic expansions (3.20) and (3.22) tell us that the pointwise estimate (3.8) is optimal. 


\section{Decay estimates for Cattaneo type heat conduction}

Now we turn to the Cattaneo case $\tau>0$, i.e we look at (1.3), (1.2) with initial data (2.6). Our purpose is also to derive optimal decay estimates for global solutions in time.

4.1. Decay estimates for $\tau>0$. Note that we have already studied (1.3), (1.2) with $a=0$ in [31]. Here we try to use similar arguments as in section 2. Introducing again $v:=u_{t}, w:=\sqrt{b} \Delta u$, our problem (1.3), (1.2) is rewritten as in the previous section as

$$
\begin{aligned}
v_{t}+\sqrt{b} \Delta w+d \Delta \theta & =0, \\
w_{t}-\sqrt{b} \Delta v & =0, \\
\theta_{t}+\operatorname{div} q-d \Delta v & =0, \\
\tau q_{t}+\kappa \nabla \psi+q & =0 .
\end{aligned}
$$

Furthermore, (4.1) with (1.2) leads

$$
\left(A_{1}^{0}+(1-a \Delta) L\right) U_{t}+\sum_{j=1}^{n} A^{j} \partial_{x_{j}} U-\Delta B U+\frac{1}{\tau}(1-a \Delta) L U=0,
$$

where $U:=(\sqrt{\kappa} v, \sqrt{\kappa} w, \sqrt{\kappa} \theta, \sqrt{\tau} q)^{T}$ and

$$
\begin{aligned}
& A_{1}^{0}:=\left(\begin{array}{cccc}
1 & 0 & 0 & 0 \\
0 & 1 & 0 & 0 \\
0 & 0 & 1 & 0 \\
0 & 0 & 0 & 0
\end{array}\right), \quad \sum_{j=1}^{n} A^{j} \xi_{j}:=\sqrt{\frac{\kappa}{\tau}}\left(\begin{array}{cccc}
0 & 0 & 0 & 0 \\
0 & 0 & 0 & 0 \\
0 & 0 & 0 & \xi \\
0 & 0 & \xi^{T} & 0
\end{array}\right), \\
& B:=\left(\begin{array}{cccc}
0 & -\sqrt{b} & -d & 0 \\
\sqrt{b} & 0 & 0 & 0 \\
d & 0 & 0 & 0 \\
0 & 0 & 0 & 0
\end{array}\right), \quad L:=\left(\begin{array}{cccc}
0 & 0 & 0 & 0 \\
0 & 0 & 0 & 0 \\
0 & 0 & 0 & 0 \\
0 & 0 & 0 & \mathrm{Id}
\end{array}\right),
\end{aligned}
$$

where $\xi=\left(\xi_{1}, \cdots, \xi_{n}\right)$. Applying the Fourier transform to $(4.2)$, we obtain

$$
\left(A_{1}^{0}+\left(1+a|\xi|^{2}\right) L\right) \hat{U}_{t}+i|\xi| A(\omega) \hat{U}+|\xi|^{2} B \hat{U}+\frac{1}{\tau}\left(1+a|\xi|^{2}\right) L \hat{U}=0
$$

where $A(\omega):=\sum_{j=1}^{n} A^{j} \omega_{j}$ for $\omega:=\xi /|\xi| \in S^{n-1}$ and $\omega=\left(\omega_{1}, \cdots, \omega_{n}\right)$. Furthermore, we introduce the new unknown function $\hat{V}:=\left(\sqrt{\kappa} \hat{v}, \sqrt{\kappa} \hat{w}, \sqrt{\kappa} \hat{\theta}, \sqrt{\tau\left(1+a|\xi|^{2}\right)} \hat{q}\right)^{T}$. Then (4.3) can be rewritten as

$$
\hat{V}_{t}+\frac{i|\xi|}{\sqrt{1+a|\xi|^{2}}} A(\omega) \hat{V}+|\xi|^{2} B \hat{V}+\frac{1}{\tau} L \hat{V}=0 .
$$

We observe that $A_{1}^{0}+L$ is the identity matrix. The solution of (4.4) can be written as

$$
\hat{V}(t, \xi)=e^{t \hat{\Phi}(i \xi)} \hat{V}_{0}(\xi)
$$


where $\hat{V}_{0}:=\left(\sqrt{\kappa} \hat{v}_{0}, \sqrt{\kappa} \hat{w}_{0}, \sqrt{\kappa} \hat{\theta}_{0}, \sqrt{\tau\left(1+a|\xi|^{2}\right)} \hat{q}_{0}\right)^{T}$ with $\hat{v}_{0}=\hat{u}_{1}$ and $\hat{w}_{0}=-\sqrt{b}|\xi|^{2} \hat{u}_{0}$, and

$$
\hat{\Phi}(i \xi):=-\left(\frac{i|\xi|}{\sqrt{1+a|\xi|^{2}}} A(\omega)+|\xi|^{2} B+\frac{1}{\tau} L\right) .
$$

Then we define the semigroup $e^{t \Phi}$ by the formula

$$
e^{t \Phi} \varphi:=\mathcal{F}^{-1}\left[e^{t \hat{\Phi}(i \xi)} \hat{\varphi}(\xi)\right] .
$$

Our main purpose is now to derive the property of the solution operator $e^{t \Phi}$.

Theorem 4.1. Let $e^{t \Phi}$ be the semigroup defined by (4.7). Then the following decay estimates hold for $1 \leq p \leq 2$ and $k \geq 0$.

$$
\left\|\partial_{x}^{k} e^{t \Phi} \varphi\right\|_{L^{2}} \leq C_{0}\left(1+\nu_{\tau, a} t\right)^{-\frac{n}{2}\left(\frac{1}{p}-\frac{1}{2}\right)-\frac{k}{2}}\|\varphi\|_{L^{p}}+C_{1}\left(1+\nu_{\tau, a} t\right)^{-\frac{\ell}{4}}\left\|\partial_{x}^{k+\ell} \varphi\right\|_{L^{2}},
$$

where

$$
\nu_{\tau, a}:=\frac{\tilde{\nu}}{(1+\tau)^{2}(1+a)},
$$

$\tilde{\nu}$ is a positive constant depending on only $b, d$ and $\kappa, C_{0}$ is a positive constant depending on only $p$ and $k$, and $C_{1}$ is a positive constant depending only on $\ell$.

From the property of the semigroup operator $e^{t \Phi}$, we derive the result on the following decay estimates for (1.3), (1.2).

Corollary 4.2. Let $(v, w, \theta, q)$ be the solution for (1.3), (1.2) with the initial data (2.6). Then the following decay estimates hold for $1 \leq p \leq 2$ and $k, \ell \geq 0$.

$$
\begin{aligned}
& \sqrt{\kappa}\left\|\partial_{x}^{k}\left(u_{t}, \Delta u, \theta\right)\right\|_{L^{2}}+\sqrt{\tau}\left\|\partial_{x}^{k} q\right\|_{H_{a}^{1}} \\
& \leq \sqrt{2} C_{0}\left(1+\nu_{\tau, a} t\right)^{-\frac{n}{2}\left(\frac{1}{p}-\frac{1}{2}\right)-\frac{k}{2}}\left(\sqrt{\kappa}\left\|\left(u_{1}, \Delta u_{0}, \theta_{0}\right)\right\|_{L^{p}}+\sqrt{\tau(1+a)}\left\|q_{0}\right\|_{L^{p}}\right) \\
& \quad+\sqrt{2} C_{1}\left(1+\nu_{\tau, a} t\right)^{-\frac{\ell}{4}}\left(\sqrt{\kappa}\left\|\partial_{x}^{k+\ell}\left(u_{1}, \Delta u_{0}, \theta_{0}\right)\right\|_{L^{2}}+\sqrt{\tau(1+a)}\left\|\partial_{x}^{k+\ell+1} q_{0}\right\|_{L^{2}}\right),
\end{aligned}
$$

where $\nu_{\tau, a}, C_{0}$ and $C_{1}$ appeared in Theorem 4.1.

In Collorary 4.2 (and Theorem 4.1 ), the loss of regularity is clearly visible: to get certain decay rates one needs more regularity of the initial data on the right-hand side than can be estimated for the solutions on the left-hand side. This is in contrast to the situation for $\tau=0$ stated in Theorem 3.1. The main estimate is the following pointwise one that will allow as conclude the rates of decay and to describe the effect of regularity loss.

The key of the proof of Theorem 3.1 is to get the pointwise estimate of the operator $e^{t \Phi}$ in Fourier space, which is stated as follows.

Proposition 4.3. Let $\hat{\Phi}(i \xi)$ be the matrix defined in (4.6). Then the corresponding matrix exponential $e^{t \hat{\Phi}(i \xi)}$ satisfies the following pointwise estimate

$$
\left|e^{t \hat{\Phi}(i \xi)}\right| \leq \sqrt{3} e^{-\frac{1}{2} \eta(\xi) t}
$$

for $t \geq 0$ and $\xi \in \mathbb{R}^{n}$, where

$$
\eta(\xi):=\frac{\tilde{\nu}|\xi|^{2}}{\left(1+\tau|\xi|^{2}\right)^{2}\left(1+a|\xi|^{2}\right)},
$$

and $\tilde{\nu}$ depends only on $b, d$ and $\kappa$. 
The precise estimate clearly describes the role of the parameters $\tau$ and $a$, leading to regularity loss whenever $\tau>0$. Formally, we recover the estimate (3.9) from section 3.

Remark 4.4. (i) The estimate (4.10) tells us that it might be better to consider not only $q$ but also the derivative of $q$ to construct the solution operator. This corresponds to the fact that we introduced $\operatorname{div} q$ in the discussion of the well-posednes in subsection 3.2.

(ii) We have the explicit value of $\tilde{\nu}$ in Proposition 4.3. The details are given in the proof of Proposition 4.3.

Proof of Proposition 4.3. We first derive the basic energy equality for the system (4.4) in the Fourier space. Taking the inner product (4.4) with $\overline{\hat{V}}$, and taking the real parts for the resulting equality, we arrive at the basic energy equality

$$
\frac{1}{2} \frac{\partial}{\partial t}|\hat{V}|^{2}+\left(1+a|\xi|^{2}\right)|\hat{q}|^{2}=0 \text {. }
$$

Here we used the symmetry properties of $A(\omega)$ and $L$, and the skew-symmetry of $B$.

Now we construct the dissipation terms. The system (4.4) is equivalent to

$$
\begin{aligned}
\hat{v}_{t}-\sqrt{b}|\xi|^{2} \hat{w}-d|\xi|^{2} \hat{\theta} & =0, \\
\hat{w}_{t}+\sqrt{b}|\xi|^{2} \hat{v} & =0, \\
\hat{\theta}_{t}-i \xi \cdot \hat{q}+d|\xi|^{2} \hat{v} & =0, \\
\tau \hat{q}_{t}+\kappa i \xi \hat{\psi}+\hat{q} & =0 .
\end{aligned}
$$

with $\hat{\theta}=\left(1+a|\xi|^{2}\right) \hat{\psi}$. We multiply the first and second equations in (4.13) by $-\overline{\hat{w}}$ and $-\overline{\hat{v}}$, respectively. Then, combining this two equations and taking real parts, we obtain

$$
-\frac{\partial}{\partial t} \operatorname{Re}(\hat{v} \overline{\hat{w}})+\sqrt{b}|\xi|^{2}\left(|\hat{w}|^{2}-|\hat{v}|^{2}\right)+d|\xi|^{2} \operatorname{Re}(\hat{w} \overline{\hat{\theta}})=0 .
$$

Furthermore, we multiply the first and the third equation in (4.13) by $d \overline{\hat{\theta}}$ and $d \overline{\hat{v}}$, respectively. Then we have

$$
d \frac{\partial}{\partial t} \operatorname{Re}(\hat{v} \overline{\hat{\theta}})+d^{2}|\xi|^{2}\left(|\hat{v}|^{2}-|\hat{\theta}|^{2}\right)+d \xi \cdot \operatorname{Re}(i \hat{v} \overline{\hat{q}})-\sqrt{b} d|\xi|^{2} \operatorname{Re}(\hat{w} \overline{\hat{\theta}})=0 .
$$

Similarly we multiply the third equation in (4.13) by $\tau i \xi \cdot \overline{\hat{q}}$ and we take the inner product of the third equation in (4.13) with $-i \xi \overline{\hat{\theta}}$. This yields

$$
\tau \xi \cdot \frac{\partial}{\partial t} \operatorname{Re}(i \hat{\theta} \overline{\hat{q}})+\kappa\left(1+a|\xi|^{2}\right)|\xi|^{2}|\hat{\psi}|^{2}-\tau|\xi \cdot \hat{q}|^{2}+\xi \cdot \operatorname{Re}(i \hat{\theta} \overline{\hat{q}})+d \tau|\xi|^{2} \xi \cdot \operatorname{Re}(i \hat{v} \overline{\hat{q}})=0 .
$$

Especially, we obtain

$$
\begin{aligned}
& \tau\left(1+a|\xi|^{2}\right) \xi \cdot \frac{\partial}{\partial t} \operatorname{Re}(i \hat{\theta} \overline{\hat{q}})+\kappa|\xi|^{2}|\hat{\theta}|^{2}-\tau\left(1+a|\xi|^{2}\right)|\xi \cdot \hat{q}|^{2} \\
& +\left(1+a|\xi|^{2}\right) \xi \cdot \operatorname{Re}(i \hat{\theta} \overline{\hat{q}})+d \tau\left(1+a|\xi|^{2}\right)|\xi|^{2} \xi \cdot \operatorname{Re}(i \hat{v} \overline{\hat{q}})=0
\end{aligned}
$$


Our next step is to combine the above equalities to construct the energy estimate. First, computing $d^{2} / 2 \times(4.14)+\sqrt{b} \times(4.15)$, we have

$$
\begin{aligned}
d \frac{\partial}{\partial t}\{ & \left.-\frac{d}{2} \operatorname{Re}(\hat{v} \overline{\hat{w}})+\sqrt{b} \operatorname{Re}(\hat{v} \overline{\hat{\theta}})\right\}+\frac{\sqrt{b} d^{2}}{2}|\xi|^{2}\left(|\hat{v}|^{2}+|\hat{w}|^{2}\right)-\sqrt{b} d^{2}|\xi|^{2}|\hat{\theta}|^{2} \\
& +\sqrt{b} d \xi \cdot \operatorname{Re}(i \hat{v} \overline{\hat{q}})+d\left(\frac{d^{2}}{2}-b\right)|\xi|^{2} \operatorname{Re}(\hat{w} \hat{\hat{\theta}})=0
\end{aligned}
$$

Here, using the Hölder inequality, we get

$$
\begin{aligned}
d \frac{\partial}{\partial t}\{ & \left.-\frac{d}{2 \sqrt{b}} \operatorname{Re}(\hat{v} \overline{\hat{w}})+\operatorname{Re}(\hat{v} \overline{\hat{\theta}})\right\}+\frac{d^{2}}{2}|\xi|^{2}|\hat{v}|^{2}+\frac{d^{2}}{4}|\xi|^{2}|\hat{w}|^{2} \\
& -\left\{d^{2}+\frac{1}{b}\left(\frac{d^{2}}{2}-b\right)^{2}\right\}|\xi|^{2}|\hat{\theta}|^{2}+d \xi \cdot \operatorname{Re}(i \hat{v} \overline{\hat{q}}) \leq 0 .
\end{aligned}
$$

On the other hand, from (4.16) we have

$$
\begin{aligned}
& \tau\left(1+a|\xi|^{2}\right) \xi \cdot \frac{\partial}{\partial t} \operatorname{Re}(i \hat{\theta} \overline{\hat{q}})+\frac{\kappa}{2}|\xi|^{2}|\hat{\theta}|^{2} \\
& -\left\{\tau|\xi|^{2}+\frac{1}{2 \kappa}\left(1+a|\xi|^{2}\right)\right\}\left(1+a|\xi|^{2}\right)|\hat{q}|^{2}+d \tau\left(1+a|\xi|^{2}\right)|\xi|^{2} \xi \cdot \operatorname{Re}(i \hat{v} \overline{\hat{q}}) \leq 0 .
\end{aligned}
$$

Then we calculate $\varepsilon_{1} \times(4.17)+(4.18)$ and obtain

$$
\begin{aligned}
& \frac{\partial}{\partial t}\left\{-\varepsilon_{1} \frac{d^{2}}{2 \sqrt{b}} \operatorname{Re}(\hat{v} \overline{\hat{w}})+\varepsilon_{1} d \operatorname{Re}(\hat{v} \overline{\hat{\theta}})+\tau\left(1+a|\xi|^{2}\right) \xi \cdot \operatorname{Re}(i \hat{\theta} \hat{\hat{q}})\right\} \\
& +\varepsilon_{1} \frac{d^{2}}{2}|\xi|^{2}|\hat{v}|^{2}+\varepsilon_{1} \frac{d^{2}}{4}|\xi|^{2}|\hat{w}|^{2}+\left\{\frac{\kappa}{2}-\varepsilon_{1}\left(d^{2}+\frac{1}{b}\left(b-\frac{d^{2}}{2}\right)^{2}\right)\right\}|\xi|^{2}|\hat{\theta}|^{2} \\
& -\left\{\tau|\xi|^{2}+\frac{1}{2 \kappa}\left(1+a|\xi|^{2}\right)\right\}\left(1+a|\xi|^{2}\right)|\hat{q}|^{2}+d\left\{\tau\left(1+a|\xi|^{2}\right)|\xi|^{2}+1\right\} \xi \cdot \operatorname{Re}(i \hat{v} \overline{\hat{q}}) \leq 0,
\end{aligned}
$$

where $\varepsilon_{1}$ is a small positive parameter. Then we put $\varepsilon_{1}=\kappa /(4 g)$, where

$$
g:=d^{2}+\frac{1}{b}\left(b-\frac{d^{2}}{2}\right)^{2}
$$

and obtain

$$
\begin{aligned}
& \frac{\partial}{\partial t}\left\{-\frac{d^{2} \kappa}{8 \sqrt{b} g} \operatorname{Re}(\hat{v} \hat{\hat{w}})+\frac{d \kappa}{4 g} \operatorname{Re}(\hat{v} \overline{\hat{\theta}})+\tau\left(1+a|\xi|^{2}\right) \xi \cdot \operatorname{Re}(i \hat{\theta} \overline{\hat{q}})\right\} \\
& +\frac{d^{2} \kappa}{8 g}|\xi|^{2}|\hat{v}|^{2}+\frac{d^{2} \kappa}{16 g}|\xi|^{2}|\hat{w}|^{2}+\frac{\kappa}{4}|\xi|^{2}|\hat{\theta}|^{2} \\
& -\left(\tau|\xi|^{2}+\frac{1}{2 \kappa}\left(1+a|\xi|^{2}\right)\right)\left(1+a|\xi|^{2}\right)|\hat{q}|^{2}+d\left\{\tau\left(1+a|\xi|^{2}\right)|\xi|^{2}+1\right\} \xi \cdot \operatorname{Re}(i \hat{v} \overline{\hat{q}}) \leq 0 .
\end{aligned}
$$

This estimate gives

$$
\begin{aligned}
& \frac{\partial}{\partial t}\left\{-\frac{d^{2} \kappa}{8 \sqrt{b} g} \operatorname{Re}(\hat{v} \overline{\hat{w}})+\frac{d \kappa}{4 g} \operatorname{Re}(\hat{v} \overline{\hat{\theta}})+\tau\left(1+a|\xi|^{2}\right) \xi \cdot \operatorname{Re}(i \hat{\theta} \overline{\hat{q}})\right\} \\
& +\frac{d^{2} \kappa}{16 g}|\xi|^{2}\left(|\hat{v}|^{2}+|\hat{w}|^{2}\right)+\frac{\kappa}{4}|\xi|^{2}|\hat{\theta}|^{2}-G(|\xi|)|\hat{q}|^{2} \leq 0 .
\end{aligned}
$$


Here, we calculate

$$
\begin{aligned}
G(|\xi|) & :=\left(\tau|\xi|^{2}+\frac{1}{2 \kappa}\left(1+a|\xi|^{2}\right)\right)\left(1+a|\xi|^{2}\right)+\frac{4 g}{\kappa}\left(\tau\left(1+a|\xi|^{2}\right)|\xi|^{2}+1\right)^{2} \\
& =\frac{4 g}{\kappa}+\frac{1}{\kappa}(\kappa+8 g) \tau|\xi|^{2}\left(1+a|\xi|^{2}\right)+\frac{1}{2 \kappa}\left(1+8 g \tau^{2}|\xi|^{4}\right)\left(1+a|\xi|^{2}\right)^{2} \\
& \leq \frac{1}{2 \kappa}\left(1+8 g+2(\kappa+8 g) \tau|\xi|^{2}+8 g \tau^{2}|\xi|^{4}\right)\left(1+a|\xi|^{2}\right)^{2} \\
& \leq \frac{1+\kappa+8 g}{2 \kappa}\left(1+\tau|\xi|^{2}\right)^{2}\left(1+a|\xi|^{2}\right)^{2} .
\end{aligned}
$$

This yields

$$
\begin{aligned}
& \frac{\partial}{\partial t}\left\{-\frac{d^{2} \kappa}{8 \sqrt{b} g} \operatorname{Re}(\hat{v} \overline{\hat{w}})+\frac{d \kappa}{4 g} \operatorname{Re}(\hat{v} \overline{\hat{\theta}})+\tau\left(1+a|\xi|^{2}\right) \xi \cdot \operatorname{Re}(i \hat{\theta} \overline{\hat{q}})\right\}+\frac{\kappa}{4}|\xi|^{2}|\hat{\theta}|^{2} \\
& +\frac{d^{2} \kappa}{16 g}|\xi|^{2}\left(|\hat{v}|^{2}+|\hat{w}|^{2}\right)-\frac{1+\kappa+8 g}{2 \kappa}\left(1+\tau|\xi|^{2}\right)^{2}\left(1+a|\xi|^{2}\right)^{2}|\hat{q}|^{2} \leq 0 .
\end{aligned}
$$

Therefore, calculating $\left(1+\tau|\xi|^{2}\right)^{2}\left(1+a|\xi|^{2}\right) \times(4.12)+\varepsilon_{0} \times(4.19)$ leads to

$$
\begin{aligned}
& \frac{\partial}{\partial t} E(t, \xi)+\varepsilon_{0} \frac{d^{2} \kappa}{16 g}|\xi|^{2}\left(|\hat{v}|^{2}+|\hat{w}|^{2}\right)+\varepsilon_{0} \frac{\kappa}{4}|\xi|^{2}|\hat{\theta}|^{2} \\
& \quad+\left(1-\varepsilon_{0} \frac{1+\kappa+8 g}{2 \kappa}\right)\left(1+\tau|\xi|^{2}\right)^{2}\left(1+a|\xi|^{2}\right)^{2}|\hat{q}|^{2} \leq 0 .
\end{aligned}
$$

where the energy $E(t, \xi)$ is defined by

$$
\begin{aligned}
E(t, \xi):= & \frac{1}{2}\left(1+\tau|\xi|^{2}\right)^{2}\left(1+a|\xi|^{2}\right)\left(\kappa\left(|\hat{v}|^{2}+|\hat{w}|^{2}+|\hat{\theta}|^{2}\right)+\tau\left(1+a|\xi|^{2}\right)|\hat{q}|^{2}\right) \\
& +\varepsilon_{0}\left(-\frac{d^{2} \kappa}{8 \sqrt{b} g} \operatorname{Re}(\hat{v} \overline{\hat{w}})+\frac{d \kappa}{4 g} \operatorname{Re}(\hat{v} \overline{\hat{\theta}})+\tau\left(1+a|\xi|^{2}\right) \xi \cdot \operatorname{Re}(i \hat{\theta} \overline{\hat{q}})\right) .
\end{aligned}
$$

Finally, we choose the positive parameter $\varepsilon_{0}$ suitably. We compute

$$
\begin{aligned}
E(t, \xi) \geq & \frac{1}{2}\left(1+\tau|\xi|^{2}\right)^{2}\left(1+a|\xi|^{2}\right)\left(\kappa\left(|\hat{v}|^{2}+|\hat{w}|^{2}+|\hat{\theta}|^{2}\right)+\tau\left(1+a|\xi|^{2}\right)|\hat{q}|^{2}\right) \\
- & \frac{\varepsilon_{0}}{2}\left(\frac{d \kappa}{4 g}\left(1+\frac{d}{2 \sqrt{b}}\right)|\hat{v}|^{2}+\frac{d^{2} \kappa}{8 \sqrt{b} g}|\hat{w}|^{2}+\left(1+\frac{d \kappa}{4 g}\right)|\hat{\theta}|^{2}+\tau^{2}|\xi|^{2}\left(1+a|\xi|^{2}\right)^{2}|\hat{q}|^{2}\right) \\
\geq & \frac{1}{2}\left(1+\tau|\xi|^{2}\right)^{2}\left(1+a|\xi|^{2}\right)\left(\kappa\left(|\hat{v}|^{2}+|\hat{w}|^{2}+|\hat{\theta}|^{2}\right)+\tau\left(1+a|\xi|^{2}\right)|\hat{q}|^{2}\right) \\
& \quad-\frac{\varepsilon_{0}}{2}\left(1+\frac{d \kappa}{4 g}\left(1+\frac{d}{2 \sqrt{b}}\right)\right)\left(|\hat{v}|^{2}+|\hat{w}|^{2}+|\hat{\theta}|^{2}+\tau^{2}|\xi|^{2}\left(1+a|\xi|^{2}\right)^{2}|\hat{q}|^{2}\right) .
\end{aligned}
$$

Thus, choosing $\varepsilon_{0}$ such that

$$
\kappa-\varepsilon_{0}\left(1+\frac{d \kappa}{4 g}\left(1+\frac{d}{2 \sqrt{b}}\right)\right) \geq \frac{\kappa}{2}, \quad 1-\varepsilon_{0}\left(1+\frac{d \kappa}{4 g}\left(1+\frac{d}{2 \sqrt{b}}\right)\right) \geq \frac{1}{2},
$$

we can obtain

$$
E(t, \xi) \geq \frac{1}{4}\left(1+\tau|\xi|^{2}\right)^{2}\left(1+a|\xi|^{2}\right)\left(\kappa\left(|\hat{v}|^{2}+|\hat{w}|^{2}+|\hat{\theta}|^{2}\right)+\tau\left(1+a|\xi|^{2}\right)|\hat{q}|^{2}\right) .
$$


Here, $\varepsilon_{0}$ should satisfy

$$
\varepsilon_{0} \leq \frac{\kappa}{2}\left(1+\frac{d \kappa}{4 g}\left(1+\frac{d}{2 \sqrt{b}}\right)\right)^{-1}, \quad \varepsilon_{0} \leq \frac{1}{2}\left(1+\frac{d \kappa}{4 g}\left(1+\frac{d}{2 \sqrt{b}}\right)\right)^{-1} .
$$

Similarly, we also compute

$$
\begin{aligned}
E(t, \xi) \leq & \frac{1}{2}\left(1+\tau|\xi|^{2}\right)^{2}\left(1+a|\xi|^{2}\right)\left(\kappa\left(|\hat{v}|^{2}+|\hat{w}|^{2}+|\hat{\theta}|^{2}\right)+\tau\left(1+a|\xi|^{2}\right)|\hat{q}|^{2}\right) \\
& \quad+\frac{\varepsilon_{0}}{2}\left(1+\frac{d \kappa}{4 g}\left(1+\frac{d}{2 \sqrt{b}}\right)\right)\left(|\hat{v}|^{2}+|\hat{w}|^{2}+|\hat{\theta}|^{2}+\tau^{2}|\xi|^{2}\left(1+a|\xi|^{2}\right)^{2}|\hat{q}|^{2}\right) \\
\leq & \frac{3}{4}\left(1+\tau|\xi|^{2}\right)^{2}\left(1+a|\xi|^{2}\right)\left(\kappa\left(|\hat{v}|^{2}+|\hat{w}|^{2}+|\hat{\theta}|^{2}\right)+\tau\left(1+a|\xi|^{2}\right)|\hat{q}|^{2}\right)
\end{aligned}
$$

for suitably small $\varepsilon_{0}$ which satisfy (4.21). Consequently, because of (4.20), we set $\varepsilon_{0}=c_{*}$ with

$$
c_{*}:=\min \left\{\frac{\kappa}{1+\kappa+8 g}, \frac{\kappa}{2}\left(1+\frac{d \kappa}{4 g}\left(1+\frac{d}{2 \sqrt{b}}\right)\right)^{-1}, \frac{1}{2}\left(1+\frac{d \kappa}{4 g}\left(1+\frac{d}{2 \sqrt{b}}\right)\right)^{-1}\right\}
$$

to obtain

$$
\begin{gathered}
\frac{\partial}{\partial t} E(t, \xi)+c_{*} \frac{d^{2} \kappa}{16 g}|\xi|^{2}\left(|\hat{v}|^{2}+|\hat{w}|^{2}\right)+c_{*} \frac{\kappa}{4}|\xi|^{2}|\hat{\theta}|^{2} \\
+\frac{1}{2}\left(1+\tau|\xi|^{2}\right)^{2}\left(1+a|\xi|^{2}\right)^{2}|\hat{q}|^{2} \leq 0
\end{gathered}
$$

and

$$
\begin{aligned}
& \frac{1}{4}\left(1+\tau|\xi|^{2}\right)^{2}\left(1+a|\xi|^{2}\right)\left(\kappa\left(|\hat{v}|^{2}+|\hat{w}|^{2}+|\hat{\theta}|^{2}\right)+\tau\left(1+a|\xi|^{2}\right)|\hat{q}|^{2}\right) \\
& \leq E(t, \xi) \leq \frac{3}{4}\left(1+\tau|\xi|^{2}\right)^{2}\left(1+a|\xi|^{2}\right)\left(\kappa\left(|\hat{v}|^{2}+|\hat{w}|^{2}+|\hat{\theta}|^{2}\right)+\tau\left(1+a|\xi|^{2}\right)|\hat{q}|^{2}\right)
\end{aligned}
$$

By using (4.22) and (4.23), we arrive at (4.10) and the energy estimate. Indeed, integrating (4.22) over $t$ and applying (4.23) to the resulting estimate, we obtain

$$
\begin{gathered}
|\hat{V}(t, \xi)|^{2}+\int_{0}^{t} \frac{c_{*} \kappa|\xi|^{2}}{\left(1+\tau|\xi|^{2}\right)^{2}\left(1+a|\xi|^{2}\right)}\left\{\frac{d^{2}}{4 g}\left(\left|\hat{v}\left(t^{\prime}, \xi\right)\right|^{2}+\left|\hat{w}\left(t^{\prime}, \xi\right)\right|^{2}\right)+\left|\hat{\theta}\left(t^{\prime}, \xi\right)\right|^{2}\right\} d t^{\prime} \\
+\int_{0}^{t} 2\left(1+a|\xi|^{2}\right)\left|\hat{q}\left(t^{\prime}, \xi\right)\right|^{2} d t^{\prime} \leq 3\left|\hat{V}_{0}(\xi)\right|^{2} .
\end{gathered}
$$

This gives

$$
\begin{aligned}
\kappa \| \partial_{x}^{k}(v, w, \theta)(t) & \left\|_{H_{\tau, a}^{3}}^{2}+\tau\right\| \partial_{x}^{k} q(t)\left\|_{H_{\tau, a}^{4}}^{2}+\frac{c_{*} d^{2} \kappa}{4 g} \int_{0}^{t}\right\| \partial_{x}^{k+1}(v, w, \theta)\left(t^{\prime}\right) \|_{L^{2}}^{2} d t^{\prime} \\
& +2 \int_{0}^{t}\left\|\partial_{x}^{k} q\left(t^{\prime}\right)\right\|_{H_{\tau, a}^{4}}^{2} d t^{\prime} \leq 3\left(\kappa\left\|\partial_{x}^{k}\left(v_{0}, w_{0}, \theta_{0}\right)\right\|_{H_{\tau, a}^{3}}^{2}+\tau\left\|\partial_{x}^{k} q_{0}\right\|_{H_{\tau, a}^{4}}^{2}\right)
\end{aligned}
$$

for $k \geq 0$. On the other hand, (4.22) gives

$$
\frac{\partial}{\partial t} E(t, \xi)+\frac{c_{*} d^{2}}{16 g}|\xi|^{2}\left\{\kappa\left(|\hat{v}|^{2}+|\hat{w}|^{2}+|\hat{\theta}|^{2}\right)+\tau\left(1+a|\xi|^{2}\right)|\hat{q}|^{2}\right\} \leq 0 .
$$


Moreover, (4.23) and the above estimate lead

$$
\frac{\partial}{\partial t} E(t, \xi)+\frac{c_{*} d^{2}}{12 g} \frac{|\xi|^{2}}{\left(1+\tau|\xi|^{2}\right)^{2}\left(1+a|\xi|^{2}\right)} E(t, \xi) \leq 0 .
$$

This yields $E(t, \xi) \leq e^{-\eta(\xi) t} E(0, \xi)$, and hence $|\hat{V}(t, \xi)| \leq \sqrt{3} e^{-\eta(\xi) t / 2}\left|\hat{V}_{0}(\xi)\right|$, where $\eta(\xi)$ is defined in (4.11) with $\tilde{\nu}:=c_{*} d^{2} /(12 g)$. Consequently, this estimate together with (4.5) gives (4.10).

As an easy consequence of Proposition 4.3, we can obtain the decay estimate for the solutions in Theorem 4.1 and Collorary 4.2.

Proof of Theorem 4.1 and Collorary 4.2. First, we prove Theorem 4.1. Because of (4.10) we have

$$
\begin{aligned}
\left\|\partial_{x}^{k} e^{t \Phi} \varphi\right\|_{L^{2}}^{2} & \leq 3 \int_{|\xi| \leq 1}|\xi|^{2 k} e^{-2 c_{0} \eta(\xi) t}|\hat{\varphi}(\xi)|^{2} d \xi+3 \int_{|\xi| \geq 1}|\xi|^{2 k} e^{-2 c_{0} \eta(\xi) t}|\hat{\varphi}(\xi)|^{2} d \xi \\
& =: I_{1}+I_{2} .
\end{aligned}
$$

For the case $|\xi| \leq 1$, we employ the same argument as in the proof of Theorem 3.1. More precisely, because of $\eta(\xi) \geq \nu_{\tau, a}|\xi|^{2}$, we get

$$
I_{1} \leq 3 \int_{|\xi| \leq 1}|\xi|^{2 k} e^{-\nu_{\tau, a}|\xi|^{2} t}|\hat{\varphi}(\xi)|^{2} d \xi \leq C_{0}^{2}\left(1+\nu_{\tau, a} t\right)^{-n\left(\frac{1}{p}-\frac{1}{2}\right)-k}\|\varphi\|_{L^{p}}^{2}
$$

for $1 \leq p \leq 2$, where $C_{0}$ is also a positive constant depending only on $p$ and $k$. On the other hand, for the case $|\xi| \geq 1$, we calculate $\eta(\xi) \geq \nu_{\tau, a}|\xi|^{-4}$ and

$$
\begin{aligned}
I_{2} & \leq 3 \int_{|\xi| \geq 1}|\xi|^{2 k} e^{-\nu_{\tau, a}|\xi|^{-4} t}|\hat{\varphi}(\xi)|^{2} d \xi \\
& \leq 3 \sup _{|\xi| \geq 1}\left\{|\xi|^{-2 \ell} e^{-\nu_{\tau, a}|\xi|^{-4} t}\right\} \int_{|\xi| \geq 1}|\xi|^{2(k+\ell)}|\hat{\varphi}(\xi)|^{2} d \xi \\
& \leq C_{1}^{2}\left(1+\nu_{\tau, a} t\right)^{-\ell / 2}\left\|\partial_{x}^{k+\ell} \varphi\right\|_{L^{2}}^{2}
\end{aligned}
$$

for $\ell \geq 0$, where $C_{1}$ is also positive constant depending only on $\ell$. Consequently, substituting the estimates of $I_{1}$ and $I_{2}$ into (4.24), we get (4.8) and Theorem 4.1 is proved.

To prove Corollary 4.2, we modify the proof of Theorem 4.1. The left-hand side of (4.24) is estimated by

$$
\begin{aligned}
\left\|\partial_{x}^{k} V(t)\right\|_{L^{2}}^{2} & \leq 3 \int_{|\xi| \leq 1}|\xi|^{2 k} e^{-\eta(\xi) t}\left|\hat{V}_{0}(\xi)\right|^{2} d \xi+3 \int_{|\xi| \geq 1}|\xi|^{2 k} e^{-\eta(\xi) t}\left|\hat{V}_{0}(\xi)\right|^{2} d \xi \\
& =: J_{1}+J_{2} .
\end{aligned}
$$

For the low frequency part, we estimate

$$
\begin{aligned}
J_{1} & \leq 3 \kappa \int_{|\xi| \leq 1}|\xi|^{2 k} e^{-\eta(\xi) t}\left|\left(\hat{v}_{0}, \hat{w}_{0}, \hat{\theta}_{0}\right)(\xi)\right|^{2} d \xi+3 \tau \int_{|\xi| \leq 1}\left(1+a|\xi|^{2}\right)|\xi|^{2 k} e^{-\eta(\xi) t}\left|\hat{q}_{0}(\xi)\right|^{2} d \xi \\
& \leq 3 \int_{|\xi| \leq 1}|\xi|^{2 k} e^{-\nu_{\tau, a}|\xi|^{2} t}\left(\kappa\left|\left(\hat{v}_{0}, \hat{w}_{0}, \hat{\theta}_{0}\right)(\xi)\right|^{2}+\tau(1+a)\left|\hat{q}_{0}(\xi)\right|^{2}\right) d \xi \\
& \leq C_{0}^{2}\left(1+\nu_{\tau, a} t\right)^{-n\left(\frac{1}{p}-\frac{1}{2}\right)-k}\left(\kappa\left\|\left(v_{0}, w_{0}, \theta_{0}\right)\right\|_{L^{q}}^{2}+\tau(1+a)\left\|q_{0}\right\|_{L^{q}}^{2}\right)
\end{aligned}
$$


for $1 \leq q \leq 2$, where $C_{0}$ is same as before. Similarly, for the high frequency part, we compute

$$
\begin{aligned}
J_{2} & \leq 3 \kappa \int_{|\xi| \geq 1}|\xi|^{2 k} e^{-\eta(\xi) t}\left|\left(\hat{v}_{0}, \hat{w}_{0}, \hat{\theta}_{0}\right)(\xi)\right|^{2} d \xi+3 \tau \int_{|\xi| \geq 1}\left(1+a|\xi|^{2}\right)|\xi|^{2 k} e^{-\eta(\xi) t}\left|\hat{q}_{0}(\xi)\right|^{2} d \xi \\
& \leq 3 \int_{|\xi| \geq 1}|\xi|^{2 k} e^{-\eta(\xi) t}\left(\kappa\left|\left(\hat{v}_{0}, \hat{w}_{0}, \hat{\theta}_{0}\right)(\xi)\right|^{2}+\tau(1+a)|\xi|^{2}\left|\hat{q}_{0}(\xi)\right|^{2}\right) d \xi \\
& \leq C_{1}^{2}\left(1+\nu_{\tau, a} t\right)^{-\ell / 2}\left(\kappa\left\|\partial_{x}^{k+\ell}\left(v_{0}, w_{0}, \theta_{0}\right)\right\|_{L^{2}}^{2}+\tau(1+a)\left\|\partial_{x}^{k+\ell+1} q_{0}\right\|_{L^{2}}^{2}\right)
\end{aligned}
$$

for $\ell \geq 0$, where $C_{1}$ is also same as before. Therefore, using

$$
\left\|\partial_{x}^{k} V(t)\right\|_{L^{2}}^{2}=\kappa\left\|\partial_{x}^{k}(v, w, \theta)(t)\right\|_{L^{2}}^{2}+\tau\left\|\partial_{x}^{k} q(t)\right\|_{H_{a}^{1}}^{2},
$$

we arrive at the desired decay estimate (4.9), and the proof is completed.

4.2. Optimality of the decay estimates $(\tau>0)$. At the rest of this section, we investigate the optimality of the estimates in Theorem 4.1 and Corollary 4.2. For this purpose we consider the characteristic equation for the system (4.1) given by

$$
\begin{aligned}
\tau\left(1+a|\xi|^{2}\right) \lambda^{4}+\left(1+a|\xi|^{2}\right) \lambda^{3}+\left\{\tau\left(b+d^{2}\right)\left(1+a|\xi|^{2}\right)|\xi|^{2}+\kappa\right\}|\xi|^{2} \lambda^{2} \\
+\left(b+d^{2}\right)\left(1+a|\xi|^{2}\right)|\xi|^{4} \lambda+b \kappa|\xi|^{6}=0 .
\end{aligned}
$$

Similarly as before, we consider the asymptotic expansion of $\lambda=\lambda(|\xi|)$ for $|\xi| \rightarrow 0$ and for $|\xi| \rightarrow \infty$. In the low frequency parts, the solutions for (4.25) are expanded as

$$
\lambda_{j}(|\xi|)=z_{j}|\xi|^{2}+O\left(|\xi|^{4}\right), \quad \lambda_{4}(|\xi|)=-\frac{1}{\tau}+\kappa|\xi|^{2}+O\left(|\xi|^{4}\right)
$$

for $j=1,2,3$, where the $z_{j}$ are solutions for $f(z)=0$ with (3.19). Observe that $\operatorname{Re}\left(z_{j}\right)<0$ for $j=1,2,3$. On the other hand, in the high frequency parts, this yields

$$
\lambda_{j}(|\xi|)= \pm \sigma i|\xi|^{2} \pm \frac{d^{2} \kappa i}{2 \sigma^{3} a \tau}|\xi|^{-2}-\frac{d^{2} \kappa}{2 \sigma^{4} a \tau}\left(\frac{1}{\tau} \pm \frac{\sigma i}{a}\right)|\xi|^{-4}+O\left(|\xi|^{-6}\right)
$$

for $j=1,2$, and

$$
\lambda_{j}(|\xi|)=-\frac{1}{2 \tau}\left(1 \pm \sqrt{1-\frac{4 b \kappa \tau}{\sigma^{2} a}}\right)+O\left(|\xi|^{-2}\right)
$$

for $j=3,4$, where $\sigma:=\sqrt{b+d^{2}}$. Especially, the expansion (4.26) gives us

$$
\operatorname{Re}\left(\lambda_{j}\right)(|\xi|)=-\frac{d^{2} \kappa}{2\left(b+d^{2}\right)^{2} a \tau^{2}}|\xi|^{-4}+O\left(|\xi|^{-6}\right)
$$

for $j=1,2$, and leads to the regularity-loss phenomena. Consequently, these asymptotic expansions tell us that the pointwise estimate (4.10) is optimal.

\section{REFERENCES}

[1] Avalos, G., Lasiecka, I.: Exponential stability of a thermoelastic system without mechanical dissipation. Rend. Instit. Mat. Univ. Trieste Suppl. 28 (1997), 1-28.

[2] Chen, P.J., Gurtin, M.E.: On a theory of heat involving two temperatures. J. Appl. Math. Phys. (ZAMP) 19 (1968), 614-627.

[3] Chen, P.J., Gurtin, M.E., Williams, W.O.: A note on non-simple heat conduction J. Appl. Math. Phys. (ZAMP) 19 (1968), 969-970. 
[4] Chen, P.J., Gurtin, M.E., Williams, W.O.: On the thermodynamics of non-simple materials with two temperatures J. Appl. Math. Phys. (ZAMP) 20 (1969), 107-112.

[5] Denk, R., Racke, R.: $L^{p}$ resolvent estimates and time decay for generalized thermoelastic plate equations, Electronic J. Differential Equations 48 (2006), 1-16.

[6] Denk, R., Racke, R., Shibata, Y.: $L_{p}$ theory for the linear thermoelastic plate equations in bounded and exterior domains. Advances Differential Equations 14 (2009), 685-715.

[7] Denk, R., Racke, R., Shibata, Y.: Local energy decay estimate of solutions to the thermoelastic plate equations in two- and three-dimensional exterior domains. J. Analysis Appl. 29 (2010), $21-62$.

[8] Fernández Sare, H.D., Muñoz Rivera, J.E.: Optimal rates of decay in 2-d thermoelasticity with second sound. J. Math. Phys. 53 (2012), 073509-1-13.

[9] Fernández Sare, H.D., Racke, R.: On the stability of damped Timoshenko systems - Cattaneo versus Fourier law. Arch. Rational Mech. Anal. 194 (2009), 221-251.

[10] Fischer, L.: Globale Lösungen zu Cauchyproblemen bei nichtlinearen thermoelastischen Plattengleichungen. Master thesis, University of Konstanz (2016).

[11] Ide, K., Haramoto, K., Kawashima, S.: Decay property of regularity-loss type for dissipative Timoshenko systems. Math. Mod. Meth. Appl. Sci. 18 (2008), 647-667.

[12] Ide, K., Kawashima, S.: Decay property of regularity-loss type and nonlinear effects for dissipative Timoshenko system. Math. Mod. Meth. Appl. Sci. 18 (2008), 1001-1025.

[13] Kim, J.U.: On the energy decay of a linear thermoelastic bar and plate. SIAM J. Math. Anal. 23 (1992), 889-899.

[14] Lasiecka, I., Pokojovy, M., Wan, X.: Global existence and exponential stability for a nonlinear thermoelastic Kirchhoff-Love plate. Nonlinear Anal.: Real World Appl. 38 (2017), 184-221.

[15] Lasiecka, I., Triggiani, R.: Two direct proofs on the analyticity of the S.C. semigroup arising in abstract thermoelastic equations. Advances Differential Equations 3 (1998), 387-416.

[16] Lasiecka, I., Triggiani, R.: Analyticity, and lack thereof, of thermo-elastic semigroups. ESAIM, Proc. 4 (1998),199-222.

[17] Lasiecka, I., Triggiani, R.: Analyticity of thermo-elastic semigroups with coupled hinged/Neumann boundary conditions. Abstract Appl. Anal. 3 (1998), 153-169.

[18] Lasiecka, I., Triggiani, R.: Analyticity of thermo-elastic semigroups with free boundary conditions. Annali Scuola Norm. Sup. Pisa 27 (1998), 457-482.

[19] Lasiecka, I., Wilke, M.: Maximal regularity and global existence of solutions to a quasilinear thermoelastic plate system. Discr. Cont. Dyn. Sys. 33 (2013), 5189-5202.

[20] Leseduarte, M.C., Quintanilla, R., Racke, R.: On (non-)exponential decay in generalized thermoelasticity with two temperatures. Appl. Math. Letters 70 (2017), 18-25.

[21] Liu, K., Liu, Z.: Exponential stability and analyticity of abstract linear thermoelastic systems. Z. angew. Math. Phys. 48 (1997), 885-904.

[22] Muñoz Rivera, J. E., Racke, R.: Smoothing properties, decay, and global existence of solutions to nonlinear coupled systems of thermoelastic type. SIAM J. Math. Anal. 26 (1995), 15471563.

[23] Muñoz Rivera, J.E., Racke, R.: Large solutions and smoothing properties for nonlinear thermoelastic systems. J. Differential Equations 127 (1996), 454-483.

[24] Quintanilla, R.: Exponential stability and uniqueness in thermoelasticity with two temperatures. Dynamics Continous, Discrete Impulsive Sys., Ser. A: Math. Anal., 11 (2004), 57-68

[25] Quintanilla, R.: On existence, structural stability, convergence and spatial behavior in thermoelasticity with two temperatures. Acta Mechanica, 168 (2004), 61-73

[26] Quintanilla, R., Racke, R.: Qualitative aspects of solutions in resonators. Arch. Mech. 60 (2008), 345-360.

[27] Quintanilla, R., Racke, R.: Addendum to: Qualitative aspects of solutions in resonators. Arch. Mech. 63 (2011), 429-435.

[28] Quintanilla, R., Racke, R.: Stability for thermoelastic plates with two temperatures. Discr. Cont. Dyn. Systems A (to appear). 
[29] Racke, R.: Thermoelasticity. Chapter 4 in: Handbook of Differential Equations. Vol. 5. Evolutionary Equations. Eds.: C.M. Dafermos, M. Pokorný. Elsevier (2009), 315-420.

[30] Racke, R.: Heat conduction in elastic systems: Fourier versus Cattaneo. Proc. International Conference on Heat Transfer, Fluid Mechanics and Thermodynamics, Skukuza, South Africa (2015), 356-360.

[31] Racke, R., Ueda, Y.: Dissipative structures for thermoelastic plate equations in $\mathbf{R}^{n}$. Advances. Differential Equations 21 (2016), 601-630.

[32] Racke, R., Ueda, Y.: Nonlinear thermoelastic plate equations - global existence and decay rates for the Cauchy problem. J. Differential Equations 263 (2017), 8138-8177.

[33] Said-Houari, B.: Decay properties of linear thermoelastic plates: Cattaneo versus Fourier law. Applicable Analysis 92 (2013), 424-440.

[34] Said-Houari, B., Kasimov, A.: Decay property of Timoshenko system in thermoelasticity. Math. Meth. Appl. Sci 35 (2012), 314-333.

[35] Ueda, Y., Duan, R., Kawashima, S.: Decay structure for symmetric hyperbolic systems with non-symmetric relaxation and its application. Arch. Rational Mech. Anal. 205 (2012), 239266.

[36] Youssef, H.M.: Theory of two-temperature-generalized thermoelasticity. IMA J. Appl. Math. 71 (2006), 383-390.

Department of Mathematics and Statistics, University of Konstanz, 78457 KonSTANZ, GERMANY

E-mail address: reinhard.racke@uni-konstanz.de

Faculty of Maritime Sciences, Kobe University, Kobe 658-0022, Japan

E-mail address: ueda@maritime.kobe-u.ac.jp 